\title{
Cloning and partial characterization of a gene in Bombyx mori homologous to a human adiponectin receptor
}

\author{
Minfeng Zhu, Keping Chen $\bowtie$, Yong Wang, Zhongjian Guo, Huijuan Yin, Qin Yao \\ and Huiqin Chen
}

\author{
Institute of Life Sciences, Jiangsu University, Zhenjiang, P. R. China
}

Received: 25 March, 2008; revised: 27 May, 2008; accepted: 07 June, 2008

available on-line: 14 June, 2008

\begin{abstract}
In this study, we report the cloning and characteristics of an adiponectin-like receptor gene from Bombyx mori (BmAdipoR) with highly conserved deduced amino-acid sequences and similar structure to the human adiponectin receptor (AdipoR). Structural analysis of the translated cDNA suggested it encoded a membrane protein with seven transmembrane domains. BmAdipoR was found to be expressed in multiple tissues and highly expressed in Malpighian tubules, fat body and testis. BmNPV (Bombyx mori nucleopolyhedrovirus) bacmid system combined with confocal microscopy revealed that BmAdipoR was targeted to the cell membrane. We also found that infection with BmNPV did not have an effect on BmAdipoR mRNA quantity in the midgut of susceptible Bombyx mori strain (306) at $48 \mathrm{~h}$, but BmAdipoR mRNA quantity increased significantly at $72 \mathrm{~h}$. We concluded that BmAdipoR gene was a membrane protein ubiquitously expressed in Bombyx mori tissues and that its expression was altered by treating with BmNPV.
\end{abstract}

Keywords: BmAdipoR, bioinformatics study, subcellular localization, real-time quantitative PCR, BmNPV

\section{INTRODUCTION}

Adiponectin, also termed Acrp30, AdipoQ, apM1 or GBP28, was identified originally by Scherer in 1995 (Scherer et al., 1995), and then by three other groups. It is generally assumed that adiponectin is secreted by the adipose tissue exclusively (Kadowaki et al., 2006). Adiponectin plays a number of beneficial roles in metabolism: improved insulin sensitivity, glucose tolerance and lipid profile (pattern of lipids in the blood), decreased inflammation and atherosclerosis. Its mechanisms of action, active forms, receptors and signaling pathways are not completely defined but seem to involve AMP-activated kinase (AMPK) and downstream acetyl-CoA carboxylase (ACC) (Yamauchi et al., 2002). Adiponectin stimulates glucose utilization and fatty acid oxidation by phosphorylating and activating AMPK (Yamauchi et al., 2002). Activation of AMPK results in phosphorylation of a variety of intracellular proteins and an increase in ATP generation (Winder \& Hardie, 1999). In addition, adiponectin increases fatty acid oxidation through inhibition of acetyl-Co A (Co-A) carboxylase and activation of malonyl-CoA decarboxylase resulting in reduced malonyl-CoA content (Tomas et al., 2002). A decrease in malonyl-CoA concentration increases the transport of long chain fatty acyl-CoA molecules into the mitochondria where they are oxidized (Ruderman et al., 1997).

Recently, two adiponectin receptors designated AdipoR1 and AdipoR2 were discovered in Kadowaki's laboratory (Yamauchi et al., 2003). AdipoR1 and R2 belong to a new family of membrane receptors predicted to contain seven transmembrane domains with their $\mathrm{N}$ termini as internal parts and their $\mathrm{C}$ termini as external parts, which is opposite

${ }^{\square}$ Corresponding author: Keping Chen, Institute of Life Sciences, Jiangsu University, \#301 Xuefu Road, Zhenjiang 212013, P. R. China; tel: 86-511-8879 1923; fax: 86-511-8879 1923; e-mail: kpchen@ujs.edu.cn

Abbreviations: AdipoR, adiponectin receptor; AMPK, AMP-activated kinase; ACC, acetyl-CoA carboxylase; ARH, arcuate hypothalamus; BmAdipoR, Bombyx mori adiponectin receptor; BmNPV, Bombyx mori nucleopolyhedrovirus; EGFP, enhanced green fluorescent protein; EST, expressed sequence tag; GPCRs, G protein-coupled receptors; hpi, hours post infection; MCS, multiple cloning sites; MOI, multiplicity of infection; TM, transmembrane. 
to the topology of all other reported G protein-coupled receptors (GPCRs). These proteins are structurally conserved from yeast to man (especially in the seven transmembrane domains) (Yamauchi et al., 2003). Interestingly, the AdipoR1 yeast homolog YOL002c plays a key role in metabolic pathways that regulate lipid metabolism such as fatty-acid oxidation (Karpichev et al., 2002). In humans and rodents, AdipoR1 and AdipoR2 are expressed ubiquitously. While AdipoR1 is abundantly expressed in skeletal muscle, AdipoR2 is predominantly expressed in the liver (Yamauchi et al., 2003). Both AdipoR1 and AdipoR2 genes are expressed in human monocytes and macrophages (Chinetti et al., 2004) and also in a primary human osteoblast cell line (Berner et al., 2004) and arcuate hypothalamus (ARH) (Kadowaki et al., 2008). In addition, AdipoR1 and AdipoR2 genes are expressed in rat and human pancreatic $\beta$ cells at levels similar to those expressed in the liver, but greater than that in muscle (Kharroubi et al., 2003). The expression of AdipoR1 and/or AdipoR2 is regulated under physiological and pathophysiological states including fasting/refeeding, obesity and insulin resistance (Tsuchida et al., 2004).

BmNPV is a nuclear polyhedrosis virus (NPV) that has caused the highest damage to Bombyx mori in tropical regions in recent years. BmNPV affects midgut epithelial cells, trachea system, hemolymph cells, fat body, etc. The nuclear of middle and inner cells of the silk gland are also sometimes invaded by this virus (Khurad et al., 2004). The processes of BmNPV infection cause many biochemical changes in larvae which respond to these biological phenomena by many changes of their metabolism to defend against pathogen invasion.

In the present study, we cloned a Bombyx mori adiponectin-like receptor gene which is closely related to human AdipoR and we provide novel evidence that BmAdipoR is expressed in multiple tissues including Malpighian tubules and silk glands. We also provide evidence that expression of the gene encoding BmAdipoR in the midgut of silkworm species 306 is increased significantly at $72 \mathrm{~h}$ by treatment with BmNPV.

\section{MATERIALS AND METHODS}

Animals. The highly susceptible silkworm strain $306($ LC50 $=4.95 \mathrm{BmNPV}$ polyhedra $/ \mathrm{ml})$ was raised in our lab (Xu et al., 2005). All larvae were raised with mulberry up to the fifth instar. Fifty newly exuviated fifth instar larvae were selected and fed with mulberry treated with $3.6 \times 10^{8} \mathrm{BmN}$ PV polyhedra/ml (BmNPV T3 strain).
Identification of the putative BmAdipoR. Human AdipoR2 (GenBank Accession No. NP 078827) sequence was used to conduct TBLASTN searches against silkworm EST database using NCBI default parameters. The obtained EST sequence with the highest identity was used to perform additional iterative BLASTN searches to obtain silkworm EST sequences for gene assembly. The cDNA sequence of BmAdipoR was obtained by EST assembly using SeqMan program of DNAstar.

Structural and phylogenetic analysis. Computer analyses (PREDICT PROTEIN, TMPRED, and TOPPRED (Claros \& von Heijne, 1994)) were used to predict the likely structure of the protein and its localization within the cell based on the deduced amino-acid sequence. The multiple protein sequence alignments were carried out using the program Clustal $\mathrm{X}$ and the parameters were auto-generated. The molecular evolutionary genetic tree through the Neighbor-joining method was constructed using MEGA3 (Kumar et al., 2004). The phylogenetic tree was tested using Bootstrap (1000 replicates; seed = 64,238 ). Pairwise deletion was selected for gaps/ missing data.

RT-PCR. Total RNA was extracted from silkworm (the fifth instar larvae) fat body, midgut, ovary, testis, Malpighian tubules, blood and silk gland using RNeasy kit. Following DNase-I treatment, first strand cDNA was synthesized by reverse transcription of $2 \mu \mathrm{g}$ of total RNA using $\mathrm{d}(\mathrm{T})_{30} \mathrm{~A} / \mathrm{G} / \mathrm{CA} / \mathrm{G} / \mathrm{C} / \mathrm{T}$ primer and $2 \mathrm{U}$ reverse transcriptase. Approximately $100 \mathrm{ng}$ of single stranded cDNA was used as template to amplify a $1116 \mathrm{bp}$ product of BmAdipoR, using the following primers (forward: 5'-ATCTAGAATGGATTGCGACGCAGGCA-3'; reverse: $5^{\prime}$ CTCGAGAAATGCCATTGATGTTGGTG-3') which synthesized the predicted sequences with $X b a \mathrm{I}$ and XhoI sites (shown in bold), respectively. RT-PCR was performed using the following program: $94^{\circ} \mathrm{C}$ for 90 s, 36 cycles of $94^{\circ} \mathrm{C}$ for $30 \mathrm{~s}, 58^{\circ} \mathrm{C}$ for $30 \mathrm{~s}$ and $72^{\circ} \mathrm{C}$ for $2 \mathrm{~min}$. The PCR products were subject to agarose gel electrophoresis and ethidium bromide staining for visualization. A Bm-actin A3 gene fragment was also amplified as a positive control, with primers actin F (5'-GGATGTCCACGTCGCACTTCA-3') and actin $\mathrm{R}$ (5'-GCGCGGCTACTCGTTCACTACC-3'). The RT-PCR product from each tissue was sequenced to confirm authenticity and used to construct recombination virus vBm-BmAdipoR-EGFP.

The generation of recombinant virus $\mathrm{vBm}$ BmAdipoR-EGFP and expression of EGFP-BmAdipoR fusion protein in BmN-4 cells. For the BmAdipoR gene, the pFas Bac HTb baculovirus transfer vector was used. The genes obtained above were inserted between XbaI and XhoI sites in the multiple cloning sites (MCS). The plasmid pFasBacHTbBmAdipoR-EGFP was used to transform competent 
DH10B cells containing helper and BmNPV bacmid. The transformed cells were added to $800 \mu \mathrm{l}$ of SOC and incubated at $37^{\circ} \mathrm{C}$ for $4 \mathrm{~h}$, and then were cultured on Luria-Bertani (LB) agar plates containing gentamicin, kanamycin, tetracycline, IPTG, and $\mathrm{X}-\mathrm{Gal}$ at $37^{\circ} \mathrm{C}$ overnight. After two rounds of color selection, white colonies containing BmAdipoR-EGFP gene were obtained and inoculated into $3 \mathrm{ml}$ of LB medium. The presence of BmAdipoR-EGFP gene in the recombinant bacmid was verified by PCR. BmN4 cells were transfected with recombinant bacmid (approx. $200 \mathrm{ng}$ ) using Cellfectin Reagent (Gibco$\mathrm{BRL})$ to produce the recombinant baculovirus $\mathrm{vBm}$ BmAdipoR-EGFP.

BmN-4 cells were infected with vBm-BmAdipoR-EGFP at a multiplicity of infection (MOI) of 5 . To test the subcellular localization of BmAdipoR, we used the Olympus Laser scanning confocal microscopy system, and identified mitochondria and nuclei by staining with Mito Tracker Red CMXRos and Hoechst 33258, respectively.

BmAdipoR mRNA quantitation in various tissues by real-time quantitative PCR. Total RNA was extracted from the silkworm fat body, midgut, ovary, testis, Malpighian tubules, blood and silk gland as described above. One microgram of total RNA was reverse transcribed using $\mathrm{d}(\mathrm{T})_{30} \mathrm{~A} / \mathrm{G} / \mathrm{C}$ $\mathrm{A} / \mathrm{G} / \mathrm{C} / \mathrm{T}$ primer and $2 \mathrm{U}$ reverse transcriptase in a $20 \mu \mathrm{l}$ reaction system. BmAdipoR mRNA and Bmactin A3 mRNA were quantified utilizing $2 \mu$ of the reverse transcription reaction (equivalent to $100 \mathrm{ng}$ single-stranded cDNA) as template in the real-time quantitative PCR. A 198-bp product for BmAdipoR cDNA corresponding to nucleotides 68-265 (GenBank Accession no. EF062308) was amplified using the following primers: forward 5'-CGACGAAGTCCTCGCAGAA-3' and reverse 5'-TCCACGACGCCTCCCATAC-3'. Similarly, a 284 bp product of silkworm Bm-actin A3 was amplified using the following primer set: forward 5'-GGATGTCCACGTCGCACTTCA-3' and reverse 5'-GCGCGGCTACTCGTTCACTACC-3'. The real-time quantitative PCR was performed on MX3000PTM Real-time PCR System. The thermal cycling profile consisted of initial denaturation at $95^{\circ} \mathrm{C}$ for $3 \mathrm{~min}$ and $40 \mathrm{cy}$ cles at $95^{\circ} \mathrm{C}$ for $30 \mathrm{~s}, 55^{\circ} \mathrm{C}$ for $1 \mathrm{~min}$, and $72^{\circ} \mathrm{C}$ for 30 $\mathrm{s}$. The relative expression level was analyzed with $2^{-\triangle \Delta} \mathrm{C}^{\mathrm{T}}$ method.

Effect of treatment with BmNPV on BmAdipoR mRNA expression. The 5th instar larvae of silkworm were treated with BmNPV at $24 \mathrm{hpi}, 48$ hpi and 72 hpi. Midguts were collected and frozen in liquid nitrogen and stored under $-70^{\circ} \mathrm{C}$ until analyzed. Total RNA was extracted using RNeasy kit. One microgram of DNase-I treated total RNA was reverse transcribed as described above. BmAdipoR mRNA and Bm-actin A3 mRNA quantities were determined by real-time quantitative RT-PCR using SYBR $^{\circledR}$ Green dye as described above. The amount of AdipoR mRNA was expressed as a proportion to Bm-actin A3 mRNA as described above and compared between 306 and NB.

\section{RESULTS}

\section{Identification of the full-length cDNA sequences}

TBLASTN searches against silkworm EST database with human AdipoR2 revealed one EST fragment (GenBank Accession No. CN211900) whose deduced amino-acid sequence has obvious sequence identity with it. After iterative BLAST searches and EST assemblies, we obtained the full-length cDNA sequence of BmAdipoR. Its putative amino acid sequence contained a HlyIII motif which was identical to that of human AdipoR2 (Fig. 1).

\section{Phylogenetic and structural analyses of the BmAdi- poR}

AdipoR proteins are characterized by a $7 \mathrm{TM}$ pass organization wholly contained within the UPF0073 motif. Comparison of the amino-acid sequence between species revealed that the predicted transmembrane regions are highly conserved especially in the TM3 (Fig. 2). Regions external to the TM domains are predicted to form intra- or extracellular loops. This shows that the invariant residues are found predominantly in the intracellular loops (Fig. 2). Outside of the TM 7 domain, the AdipoR proteins have distal $\mathrm{N}$ - and $\mathrm{C}$-terminal regions. The $\mathrm{N}$-terminal regions have various lengths, with highly conserved sequences near the TM domain, while the C-terminal regions have similar lengths, with relatively low sequence homologies (Fig. 3). However, a clear pattern of invariant residues $\left(\mathrm{HGXSX}_{5} \mathrm{RX}_{6} \mathrm{C}\right.$ ) within the C-terminal regions can be seen in this alignment (Fig. 2). The results of the phylogenetic analysis indicated that four major clades of AdipoRs, vertebrate, invertebrate (insects), invertebrate (Nematode), and Fungi, exist in vertebrate and invertebrate classes (Fig. 4). The BmAdipoR is classified into the invertebrate (insects) subgroup. Sequence identity comparisons also showed that BmAdipoR had a higher homology with the invertebrate (insects) group compared with other groups (not shown).

A proposed model for the insertion of the putative BmAdipoR in the plasma membrane based on hydrophobicity and charges of the amino acids analyzed by TopPred (Claros \& von Heijne, 1994) is shown in Fig. 5. It is shown that the N-terminal ends of BmAdipoR are localized intracellularly while their 
agagttcagtaggtaggtaccacaacgacacgacagtcagtaagttttacagctgtaggaatc gcacctctgcatagtaactgacgatagttcattcagtg tatadattttagctagtggtctg tcgagtgattttatcgacaaaacaacacatcagagtcattgggtgtaatttttgtgacg taattgactttgatttcgatactgttcgtgtaccgctaacaaggtcacggcggccatggt tgccaatttcccatcttcgcgtgattgaacaagcgaagatgtgggagtggatctgatattggat cccaagegtgtcctcog

1 atggattgcgacgcaggcaaggatgggatccogatgcggagagcettgcetctcaatgg

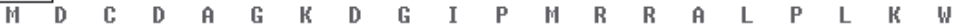

61 acgagctcgacgaagtcctcgcagaagagaagaggatgtccgctgccttcaacaccgg $\begin{array}{llllllllllllllllllll}\mathbf{T} & \mathbf{S} & \mathbf{S} & \mathbf{T} & \mathbf{K} & \mathrm{S} & \mathrm{S} & \mathbf{Q} & \mathbf{K} & \mathbf{K} & \mathbf{K} & \mathbf{K} & \mathbf{D} & \mathbf{U} & \mathbf{R} & \mathbf{C} & \mathbf{L} & \mathbf{Q} & \mathbf{H} & \mathbf{R}\end{array}$

121 aggatcacatctgttggatgccgaatggctgagttcttgaagctggcgtgttatcc $\begin{array}{llllllllllllllllllll}\mathbf{R} & \mathbf{I} & \mathbf{N} & \mathbf{I} & \mathbf{C} & \boldsymbol{W} & \mathbf{H} & \mathbf{P} & \mathbf{K} & \boldsymbol{W} & \mathrm{L} & \mathbf{K} & \mathbf{F} & \mathbf{L} & \mathbf{K} & \mathbf{A} & \mathbf{G} & \mathbf{U} & \mathbf{L} & \mathrm{S}\end{array}$

181 gatgagattgatttgggagcactggcccataacgccgccgacaggccgaggattcgtt $\begin{array}{llllllllllllllllllll}D & E & I & D & L & G & A & L & A & H & N & A & A & E & \mathbf{Q} & A & E & E & F & U\end{array}$

241 cgcaagtatgggaggcgtcgtggacgtgtgccattcagacatttgccacgttggcta $\begin{array}{llllllllllllllllllll}\mathbf{R} & \mathbf{K} & \boldsymbol{U} & \mathbf{W} & \mathbf{E} & \mathbf{A} & \mathrm{S} & \mathbf{W} & \mathbf{N} & \mathbf{U} & \mathbf{C} & \mathbf{H} & \mathbf{F} & \mathbf{R} & \mathbf{H} & \mathbf{L} & \mathbf{P} & \mathbf{R} & \mathbf{W} & \mathbf{L}\end{array}$

301 cagacaacgattatttacacaaggacatagaccgctctaccttcgttcagcgcatgt $\begin{array}{llllllllllllllllllll}\mathbf{Q} & \mathbf{D} & \mathbf{N} & \mathbf{D} & \mathbf{Y} & \mathbf{L} & \mathbf{H} & \mathbf{K} & \mathbf{G} & \mathbf{H} & \mathbf{R} & \mathbf{P} & \mathbf{P} & \mathbf{L} & \mathbf{P} & \mathbf{S} & \mathbf{F} & \mathbf{S} & \mathbf{A} & \mathbf{C}\end{array}$

361 tttgcatcatttccgaatccacactgagacaggtaacatttggacgcatctccttggc

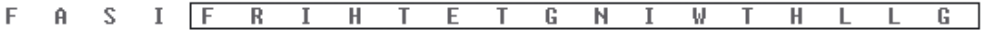

421 tgcgtggcattcattggcgtcgcgatctacttctgtctcgtccatctatcgaattcaa \begin{tabular}{|llllllllllllllllllll|}
\hline $\mathbf{C}$ & $\mathbf{U}$ & $\mathbf{A}$ & $\mathbf{F}$ & $\mathbf{I}$ & $\mathbf{G}$ & $\mathbf{U}$ & $\mathbf{A}$ & $\mathbf{I}$ & $\mathbf{Y}$ & $\mathbf{F}$ & $\mathrm{L}$ & $\mathrm{S}$ & $\mathbf{R}$ & $\mathbf{P}$ & $\mathrm{S}$ & $\mathbf{I}$ & $\mathbf{E}$ & $\mathbf{I}$ & $\mathbf{Q}$ \\
\hline
\end{tabular}

481 atgcaagagaagttatattcggtgttttttgtcggcgctatcgtatgcctcggttt \begin{tabular}{|llllllllllllllllllll|}
\hline$H$ & Q & E & K & U & I & F & G & U & F & F & U & G & A & I & U & C & L & G & F \\
\hline
\end{tabular}

541 tctttgcctatcacacactgtactgccactccgagatggtcggaagctgttctcaag \begin{tabular}{|llllllllllllllllllll|}
\hline$S$ & F & A & Y & H & T & L & Y & C & H & S & E & M & U & G & K & L & F & S & K \\
\hline
\end{tabular}

601 ctggattattgtggatagcattgctcatcatgggctccttgttccatggttgtactac \begin{tabular}{|llllllllllllllllllll|}
\hline & D & Y & C & G & I & A & L & L & I & M & G & S & F & U & P & W & L & Y & $Y$ \\
\hline
\end{tabular}

661 agtttctactgccactacagaccgagatcatatacctatctgtagtagttgtttagga

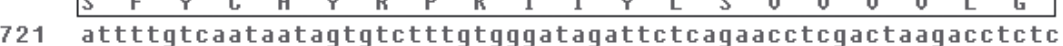
\begin{tabular}{|llllllllllllllllllll|}
\hline $\mathrm{I}$ & $\mathrm{L}$ & $\mathrm{S}$ & $\mathrm{I}$ & $\mathrm{I}$ & $\mathrm{U}$ & $\mathrm{S}$ & $\mathrm{L}$ & $\mathbf{W}$ & $\mathrm{D}$ & $\mathbf{R}$ & $\mathrm{F}$ & $\mathrm{S}$ & $\mathrm{E}$ & $\mathbf{P}$ & $\mathbf{R}$ & $\mathbf{L}$ & $\mathbf{R}$ & $\mathbf{P}$ & $\mathrm{L}$ \\
\hline
\end{tabular}

781 agagcaggagttttatgggctttggttgtctggtatagtcccagcattcactatggg \begin{tabular}{|llllllllllllllllllll}
\hline$R$ & A & G & U & F & H & G & F & G & L & S & G & I & U & P & A & I & H & Y & G \\
\hline
\end{tabular}

841 attaccgaggctggttcagtcagtcagcaagcatcattgggctggttagtttgatg \begin{tabular}{|lllllllllllllllllllll}
\hline $\mathrm{I}$ & $\mathrm{T}$ & $\mathrm{E}$ & $\mathbf{G}$ & $\mathbf{W}$ & $\mathrm{F}$ & $\mathrm{S}$ & $\mathbf{Q}$ & $\mathrm{U}$ & $\mathrm{S}$ & $\mathrm{K}$ & $\mathbf{A}$ & $\mathrm{S}$ & $\mathrm{L}$ & $\mathbf{G}$ & $\mathbf{W}$ & $\mathrm{L}$ & $\mathrm{U}$ & $\mathrm{L}$ & H \\
\hline
\end{tabular}

901 ggattgctctatatcttaggtgccatgttctatgccttagagtgccagaacttggttc \begin{tabular}{|llllllllllllllllllll|}
\hline $\mathbf{G}$ & $\mathrm{L}$ & $\mathrm{L}$ & $\mathbf{Y}$ & $\mathrm{I}$ & $\mathrm{L}$ & $\mathbf{G}$ & $\mathbf{A}$ & $\mathbf{M}$ & $\mathrm{F}$ & $\mathbf{Y}$ & $\mathbf{A}$ & $\mathrm{L}$ & $\mathbf{R}$ & $\mathbf{U}$ & $\mathbf{P}$ & $\mathrm{E}$ & $\mathbf{R}$ & $\mathbf{W}$ & $\mathrm{F}$ \\
\hline
\end{tabular}

961 cctggcaatgtgatattggttcagtcccatcaatattccatgttcttgtgattgta \begin{tabular}{|llllllllllllllllllll|}
\hline P & G & K & C & D & I & W & F & Q & S & H & Q & I & F & H & U & L & U & I & U \\
\hline
\end{tabular}

1021 gctgctttgtacattaccacggtatagcgattggcatcttacagagtcacagtagga

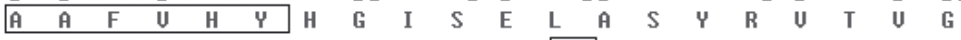

1081 gagtgctccatgccaccaacatcaatggcatttegt tacagttagaccaaatgtgatagt $\begin{array}{llllllllllll}E & C & S & \text { H } & P & P & T & S & \text { M } & \boldsymbol{A} & \mathrm{F}\end{array}$

agaccatataattaccatattacaatacagaacatctgttagttgcactgtacatattata gagtgtcattaatgaagttactggtattgtacattttattgagaaaaaatccatagtca atctgacattettraatctgttagatttgatacatgtactgaattacaaacagttcacaa ttggatctagattgatggtaataataattctgttaaaattagaatgttgttattgtagattaa agatcacagaaacattgtatgcagtttagagttttggtgttcattgttaattgaataattgt

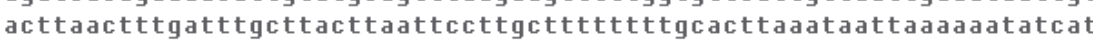
tgtaggtcagaccatagtaaatccattatgccgtttataaataaatgcttgattaatat aaaaaccttttaattattactaacacatacacaaaataattattttaagacatgt ttattcatattcaattgatctcacatttacacgttcacatattattcattccaatggt attactgatattgatt attaatgtggaatttcatatttcttatggttettacattgtaat aataatttacattaactttutgatgttgatacattccattttt attcatatcatagatt cattataccatgaagaacagtgataatgtaaaaa

Figure 1. Nucleotide sequence and deduced amino-acid sequence of the BmAdipoR.

The predicted amino acid is represented by the one letter code designation below the nucleotide sequence. The TATA-box is in italics and underlined. PolyA signals are underlined. In the amino-acid sequence, the conservative amino-acids (HlyIII) are framed.

C-terminal ends reside in the extracellular domain, which is in contrast to the GPCRs model.

\section{Expression of BmAdipoR in various tissues}

RT-PCR analysis revealed that BmAdipoR mRNAs were distributed in a wide range of Bombyx mori tissues (Fig. 6). Real-time quantitative PCR analysis showed that BmAdipoR mRNA was the highest in Malpighian tubules, fat body and testis, followed by ovary, blood, silk gland and midgut
(Fig. 6). Melting curve analyses showed the presence of a single PCR product for BmAdipoR or Bm-actin A3, confirming the specificity of the reaction (not shown).

\section{Expression of EGFP-BmAdipoR fusion protein in $\mathrm{BmN}-4$ cells}

Previous studies revealed that AdipoR is a membrane protein (Yamauchi et al., 2003). To characterize BmAdipoR, the gene was inserted into an ex- 


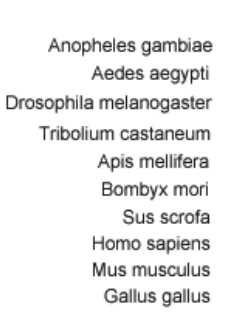

Anopheles gambiae
Aedes aegypti
Tribolium castaneum
Apis mellifera
Bombyx mori
Sus scrofa
Homo sapiens
Mus musculus
Gallus gallus

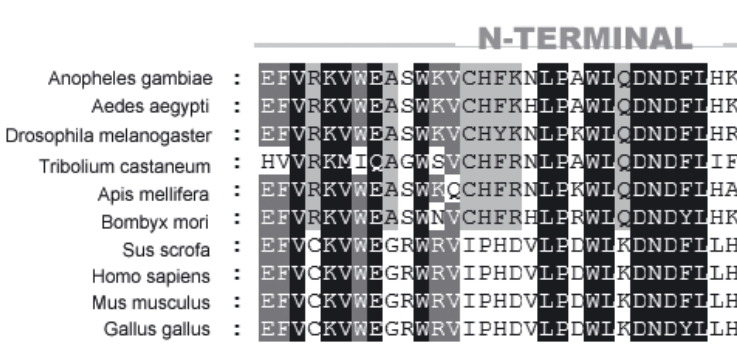

TM2

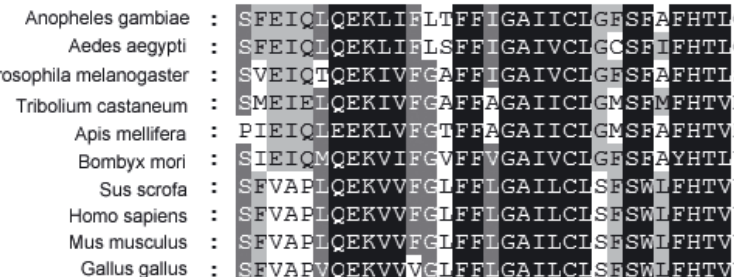

TM4
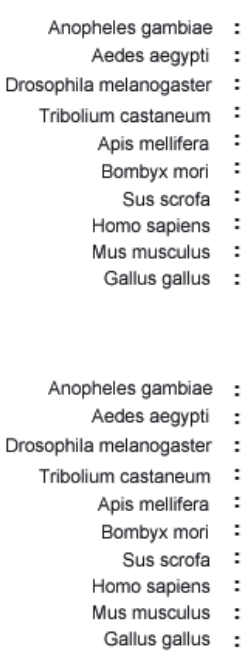

TM7
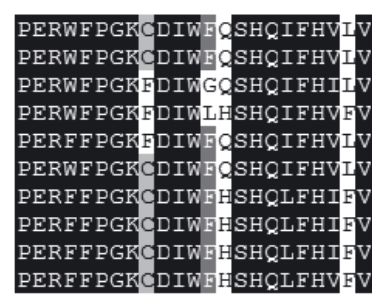

N-TERMINAL

-MSSRYDLNAPVPGMAPVTI PRETRR LDELFNNMMEADFS MDSATNLLEQQGSAADVSGGSHPAEVEVTTQARATFGMDAEGHATGEAVTTTTATLRREGSDEDIFE---QVQMI -MSHYSDAEDSID--IAADMGVRK

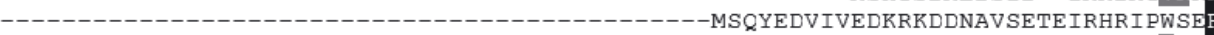
$-M D C D A G K D-$

-MNETAENRSEYSRTPEPGIF -MNEPTENRLGCSRTPEPD -MNEPAKHRLGCTRTPEPD -MNELTE--LDNAGSPEPGLE

\section{N-TERMINAL}

VWAP-EEVSLASEDIDLLDDDDD-LEEEEDDGVGCPLPSTPEDNQLLEAEMTEVLKACVIS--DEIDLGAIAHNAAEQAE : 95 VWSPEEDDSLASEDMDLLDDDDE-LEEEEDDGVGCPLPSIPEDTQLLEAEMTEVLKAGVIS--DEIDLGAIAHNAAEQAE : 123 GWGP--EDSLSPNDLDILEYDDE-LVEEDD--AGCPLPSIPEDTQLIEAEMTEVLKAGVIS--DEIDLGAIAHNAAEQAE : 150 IWDP-ETESITSEIKELLDDDVN-SLVEED-CVVGCPLPSIPEDENMLDSEMSDVLKAAVIGTGDEIDITTIAHNAASQAE : 100 LLNS--EVPGLHEVKELLEDDDASCLAEEEDGVGCPLPSIPEDDHLLDCEMTEVLKAEVIS--DEIDLGAIAHNAAEQAE : 110 ALPLKWTSSTKSSOKKKKD-------------VRCLQHRRIN--ICWMPKWLKFLKAGVISS-DEIDLGAIAHNAAEQA : 77 GHQPD-GTRRGDNDIHQGALDPV----IEATVISSCHKKSIEEPECDNEASQE--DEGHMG----NSPLICAHHAMERME : 92 GHQLD-GTRRGDNDSHQGDLEPI----LEASVIISSHHKKS SEEHEYSDEAPQE--DEG FMG----NSPLL QAHHAMEKME : 92 GHQLD-DTRGSNNDNYQGDLEPS----LETPVCSSYYEN SPEEPECHDDNSQE--DEGEMG----NSPLLQAHHAMERME : 92 GHMSDSATTQTAFEEDSSEQRLL----LVEPPIIISSNQENSSEDSNHNDESPQE--DEGFM

\section{TM1}
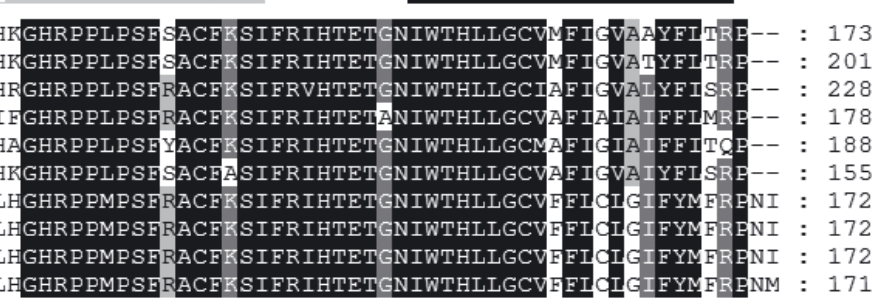

TM3

TM4
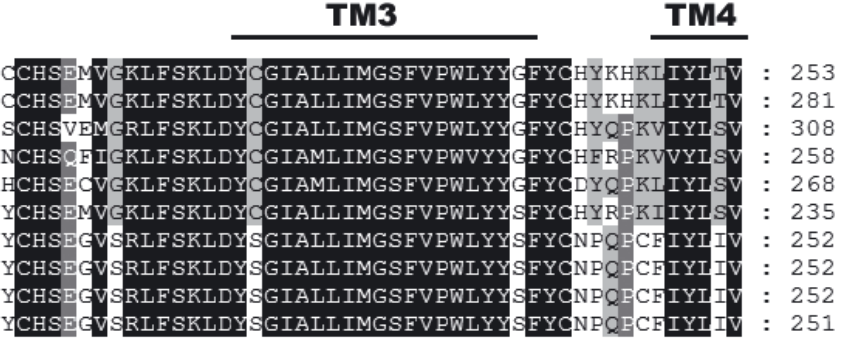

TM5

TM6
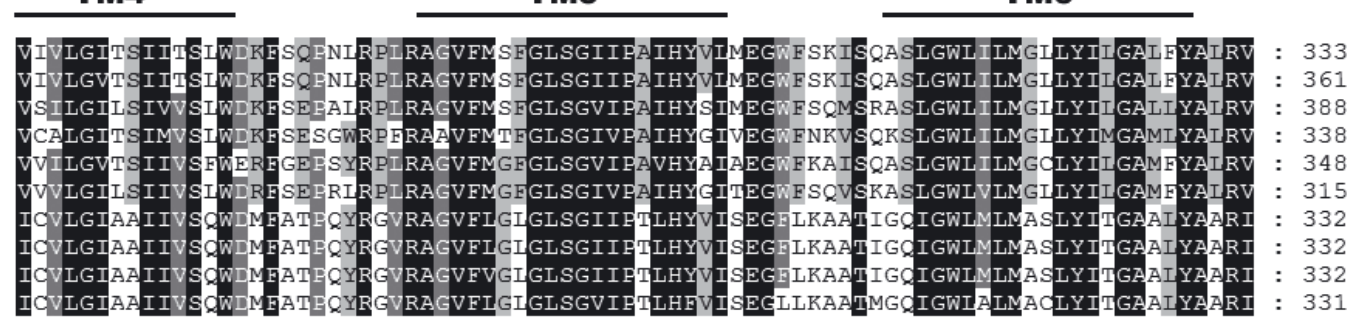

\section{C-TERMINAL}
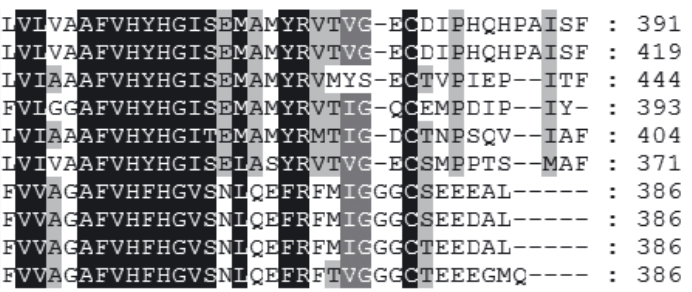

Figure 2. The multiple sequence alignments of BmAdipoR with those of other species.

Numbers on the right indicate the amino-acid position of different sequences. Identical amino acids are shaded in black. Black bars over amino-acid residues indicate the seven transmembrane domain regions as predicted by TMPRED. Gray bars over amino-acid residues indicate the distal $\mathrm{N}$ - and C-terminal regions. 


\begin{tabular}{lcccc} 
Anopheles gambiae & $\mathbf{1 4 2}$ & $\mathbf{2 2 2}$ & $\mathbf{2 7}$ & 391 \\
Aedes aegypti & $\mathbf{1 7 0}$ & $\mathbf{2 2 2}$ & $\mathbf{2 7}$ & 419 \\
Drosophila melanogaster & $\mathbf{1 9 7}$ & $\mathbf{2 2 2}$ & $\mathbf{2 5}$ & 444 \\
\cline { 2 - 5 } & $\mathbf{1 5 7}$ & $\mathbf{2 2 2}$ & $\mathbf{2 5}$ & 404 \\
Apis mellifera & $\mathbf{1 4 7}$ & $\mathbf{2 2 2}$ & $\mathbf{2 4}$ & 393 \\
Tribolium castaneum & $\mathbf{1 3 8}$ & $\mathbf{2 2 4}$ & $\mathbf{2 4}$ & 386 \\
Gallus gallus & $\mathbf{1 3 9}$ & $\mathbf{2 2 4}$ & $\mathbf{2 3}$ & 386 \\
Sus scrofa & $\mathbf{1 3 9}$ & $\mathbf{2 2 4}$ & $\mathbf{2 3}$ & 386 \\
Homo sapiens & $\mathbf{1 3 9}$ & $\mathbf{2 2 4}$ & $\mathbf{2 3}$ & 386 \\
Mus musculus & $\mathbf{1 2 4}$ & $\mathbf{2 2 2}$ & $\mathbf{2 5}$ & 371 \\
Bombyx mori & & & $\mathbf{2}$ &
\end{tabular}

Figure 3. Relative sizes of AdipoR in different species are indicated.

Each AdipoR protein encodes a predicted intracellular $\mathrm{N}$-terminal domain, a 7TM spanning sequence (encoded within the UPF0073 Pfam domain), and a predicted extracellular C-terminal domain.

pression vector that allows the production of EGFP or BmAdipoR-EGFP fusion protein. vBm-BmAdipoR-EGFP plasmids were transfected into BmN-4 cells. Localization of BmAdipoR protein was tested by fluorescence confocal microscopy. It was shown that EGFP protein was distributed throughout the entire cell in both cytoplasm and nucleus. However, BmAdipoR-EGFP was located in the cell membrane (Fig. 7).

\section{Effect of treatment with BmNPV on BmAdipoR mRNA quantity}

BmAdipoR mRNA quantity did not change significantly at $48 \mathrm{~h}$ in strain 306 midgut infected with BmNPV, but increased significantly at $72 \mathrm{~h}$ (Fig. 8). Melting curve analyses showed the presence of a single PCR product for BmAdipoR or Bm-actin $\mathrm{A} 3$, confirming the specificity of the reaction (not shown).

\section{DISCUSSION}

We have cloned and partly characterized a new adiponectin-like receptor in Bombyx mori that shares significant sequence identity with human AdipoR. By comparing BmAdipoR with AdipoRs in other animals, we found that BmAdipoR has highly conserved transmembrane regions. Structure analysis showed that the invariant residues are found predominantly in the intracellular loops or the in-

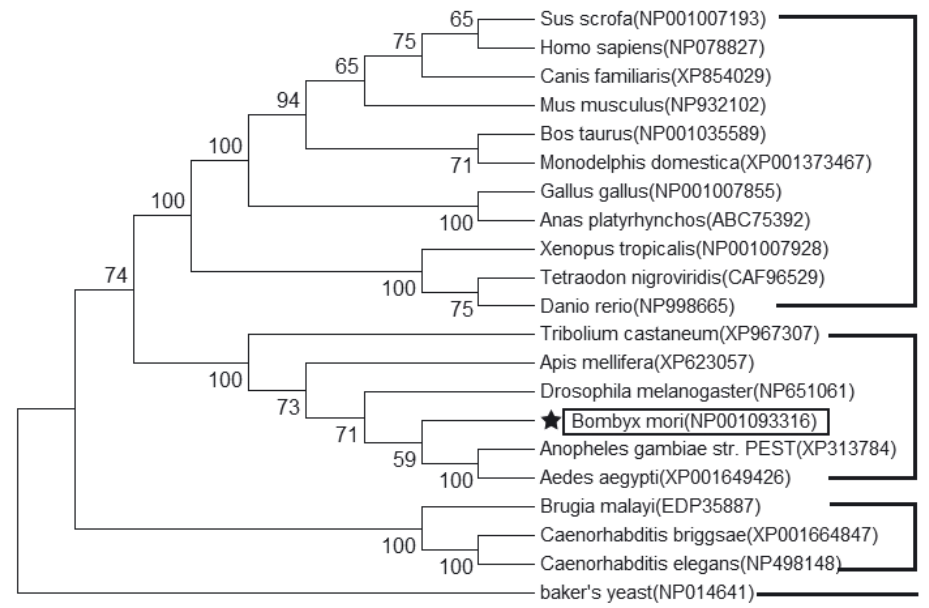

Vertebrates
Invertebrates (Insects)

Invertebrates (Nematode)

Fungi
Figure 4. Phylogenetic analysis of BmAdipoR with other AdipoR family members.

NJ tree was produced by MEGA3 which shows the four indicated clades of AdipoR. One thousand bootstraps were performed for checking reproducibility of the result.

Figure 5. Topological model for BmAdipoR protein.

Transmembrane spanning regions (Segments) were predicted using TopPred (Claros \& von Heijne, 1994). Loop length and number of Lys and Arg are indicated. 


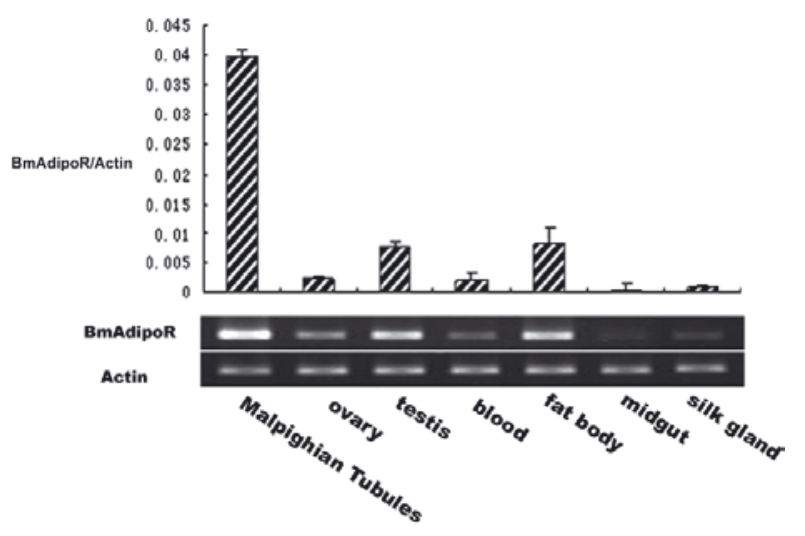

Figure 6. The relative levels of BmAdipoR from various tissues of Bombyx mori were measured by semi-quantitative RT-PCR and real-time quantitative PCR.

Using either method, the highest concentration of BmAdipoR was found in Malpighian tubules.

tracellular face of the TM domains, indicating that conserved features may be required for signal transduction and that the situation may be similar to that observed in GPCRs; for example, the rhodopsin-type GPCRs have relatively low sequence similarities, yet they maintain a 7TM architecture with invariant residues observed within the intracellular loops and much less conservation in the extracellular loops (Gether, 2000). The conserved sequences of Nterminal regions and the invariant residues (HGX$\left.\mathrm{SX}_{5} \mathrm{RX}_{6} \mathrm{C}\right)$ within the C-terminal regions may be the ligand binding sites. Phylogenetic comparisons of the deduced amino-acid sequence of BmAdipoR with homologous sequences from a variety of other species showed that BmAdipoR is clustered in the subgroup of invertebrates (insects) (Fig. 4). Computer analysis using a program that predicts protein structure (TopPred) indicates that the deduced protein is located in the plasma membrane and typically has seven transmembrane domains as well as intracellular N-terminal and extracellular C-terminal domains. This protein structure is similar to those of mammalian AdipoR1 and AdipoR2 which are opposite to the topology of all other reported GPCRs.

RT-PCR showed that BmAdipoR was expressed in multiple tissues in Bombyx mori, this pattern was similar to that of mammalian AdipoR that is expressed ubiquitously. We determined the relative expression of BmAdipoR mRNA in various tissues using real-time quantitative PCR and found that Malpighian Tubules, fat body and testis were the principal organs where BmAdipoR gene was maximally expressed. In most insects the fat body serves as a storage depot for food reserves. In addition to its important roles as a storage depot, the fat body of insects functions as a key center of metabolism and biochemistry. The BmAdipoR highly expressed in Bombyx mori fat body may possibly be involved in lipid metabolism and glucose utilization. It is tempting to speculate that BmAdipoR mRNA expressed in the testis was possibly involved in steroid synthesis as it was recently shown that AMPK, a molecule that is activated by adiponectin, is involved in progesterone synthesis
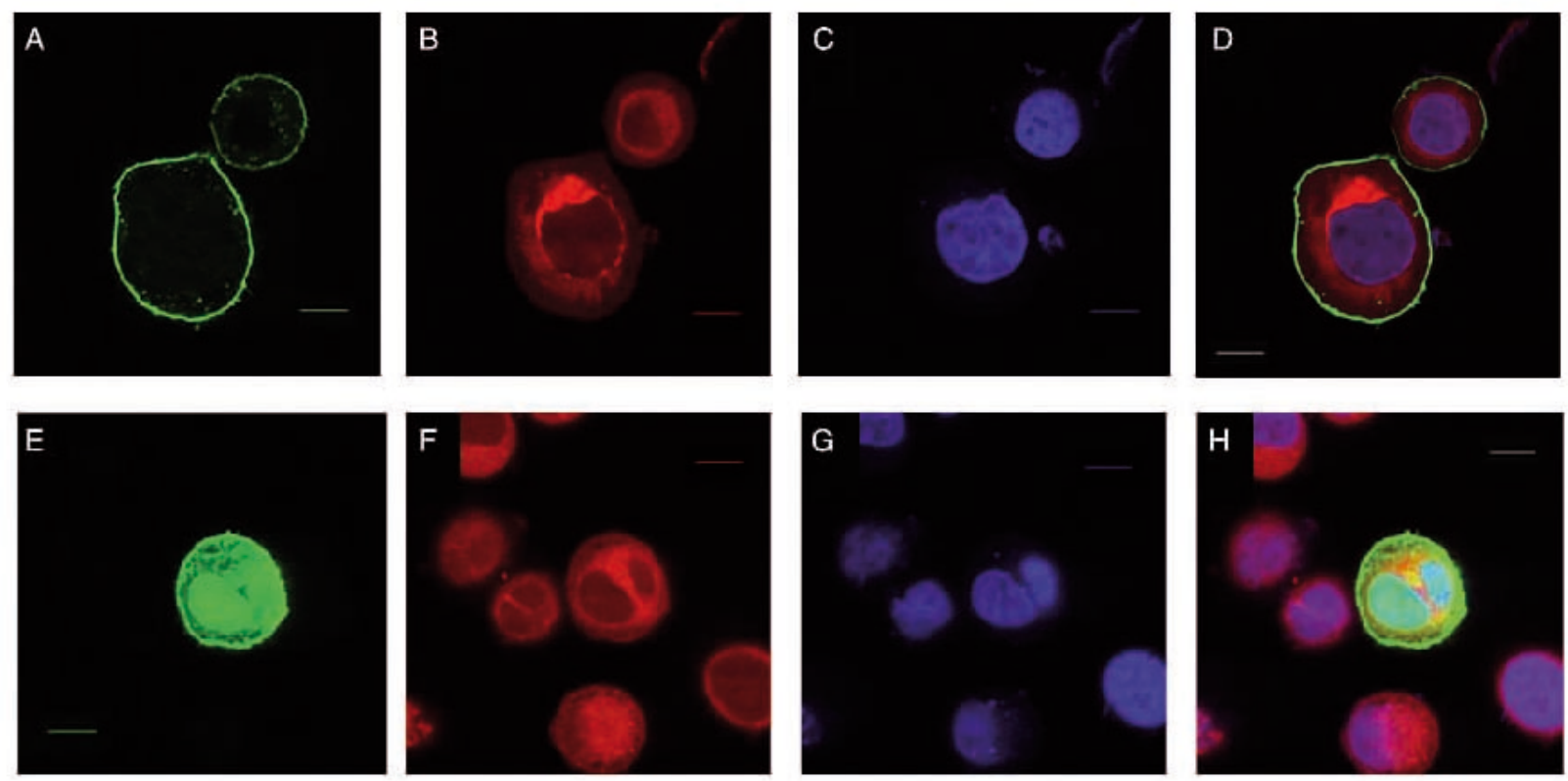

Figure 7. Cellular localization of BmAdipoR within transfected cells.

A. The cells transfected with vBm-BmAdipoR-EGFP plasmids; E. The cells transfected with vBm-EGFP plasmids; B and F. Mitochondria were stained red with Mito Tracker Red CMXRos; C and G. Nuclei were stained blue with Hoechst 33258; D. Merged images of panel A, B, and C; H. Merged images of panel E, F, and G. Bar $=10 \mu \mathrm{m}$. 


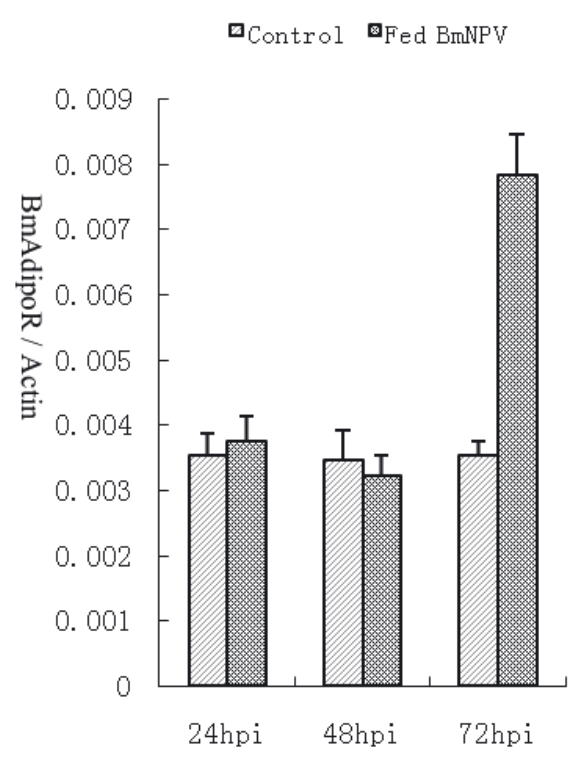

Figure 8. Effect of feed BmNPV on BmAdipoR mRNA quantity in the 306 midgut.

The 5th instar silkworm larva was reared on fresh mulberry leaves without any treatment (control). Treatment group were fed with mulberry treated with $3.6 \times 10^{8} \mathrm{Bm}-$ NPV polyhedra/ml (BmNPV T3 strain). hpi: hours post-infection.

(Tosca et al., 2005), and the rapid actions of progestins can increase sperm motility and fertilization (Thomas et al., 2005).

This is the first report describing robust expression of AdipoR mRNA in the Malpighian tubules. Good osmoregulation is critical to the success of insects, and the Malpighian tubules play a key role in osmoregulation. Recently, the application of genetics and genomics to the Drosophila tubule has revealed far more extensive roles than ion and water transport. Microarray analysis shows that organic solute transporters dominate the tubule transcriptome (Wang et al., 2004). The tubule thus has the capability to excrete actively a broad range of organic solutes and xenobiotics. Such transporters can produce unexpected, emergent roles for the whole tissue. Reinforcing this role in excretion, the tubule expresses very high levels of a particular cytochrome P450 s, glutathione-S-transferases and alcohol dehydrogenases, which suggests that the tubule plays a major role in metabolism and detoxification of both endogenous solutes and xenobiotics, such as insecticides (Dow \& Davies, 2006). The function of BmAdipoR in Bombyx mori Malpighian tubules is not clear. It may be involved in lipid metabolism of organic solutes, making toxic compounds less harmful, or at least easier to excrete.

Subcellular localization of proteins is particularly helpful in the functional annotation of gene products. In this study, we used the BmNPV bacmid system to examine the subcellular localization of BmAdipoR. The result showed that BmAdipoR was a membrane protein, which is consistent with previous studies (Yamauchi et al., 2003). This further confirmed that BmAdipoR may have the same function as mammalian AdipoR.

To see whether the BmAdipoR was involved in BmNPV infection, we investigated the effect of BmNPV infection on the expression of BmAdipoR mRNA in Bombyx mori midguts. In the present study, we found that BmAdipoR mRNA quantity did not change in strain 306 infected with BmNPV at $48 \mathrm{~h}$, but increased significantly at $72 \mathrm{~h}$. Previous studies showed that infection with BmNPV did not have a considerable effect on carbohydrates up to the third day but with time, the amount of carbohydrates was enhanced in infected Bombyx mori (Rami reddy et al., 1992). A significant increase in BmAdipoR mRNA quantity at $72 \mathrm{~h}$ in response to treatment with BmNPV may enhance the ability of adiponectin to stimulate glucose uptake. In this regard, the increased carbohydrates in larvae treated with BmNPV were at least partly caused by the increased BmAdipoR. Nevertheless, the reasons for the significant change in BmAdipoR mRNA quantity in response to treatment with BmNPV require further studies.

\section{Acknowledgements}

This work was supported by a grant from Academy Natural Science Foundation of Jiangsu Province, China (BK2006074) and National Basic Research Program of China (2005CB121000).

\section{REFERENCES}

Berner HS, Lyngstadaas SP, Spahr A, Monjo M, Thommesen L, Drevon CA, Syversen U, Reseland JE (2004) Adiponectin and its receptors are expressed in boneforming cells. Bone 35: 842-849.

Chinetti G, Zawadski C, Fruchart JC, Staels B (2004) Expression of adiponectin receptors in human macrophages and regulation by agonists of the nuclear receptors PPARalpha, PPARgamma, and LXR. Biochem Biophys Res Commun 314: 151-158.

Claros MG, von Heijne G (1994) TopPred II: an improved software for membrane protein structure predictions. Comput Appl Biosci 10: 685-686.

Dow JA, Davies SA (2006) The Malpighian tubule: rapid insights from post-genomic biology. J Insect Physiol 52: 365-378.

Gether U (2000) Uncovering molecular mechanisms involved in activation of $G$ protein-coupled receptors. Endocr Rev 21: 90-113.

Kadowaki T, Yamauchi T, Kubota N (2008) The physiological and pathophysiological role of adiponectin and adiponectin receptors in the peripheral tissues and CNS. FEBS Lett 582: 74-80.

Kadowaki T, Yamauchi T, Kubota N, Hara K, Ueki K, Tobe K (2006) Adiponectin and adiponectin receptors 
in insulin resistance, diabetes, and the metabolic syndrome. J Clin Invest 116: 1784-1792.

Karpichev IV, Cornivelli L, Small GM (2002) Multiple regulatory roles of a novel Saccharomyces cerevisiae protein, encoded by YOL002c, in lipid and phosphate metabolism. J Biol Chem 277: 19609-19617.

Kharroubi I, Rasschaert J, Eizirik DL, Cnop M (2003) Expression of adiponectin receptors in pancreatic beta cells. Biochem Biophys Res Commun 312: 1118-1122.

Khurad AM, Mahulikar A, Rathod MK, Rai MM, Kanginakudru S, Nagaraju J (2004) Vertical transmission of nucleopolyhedrovirus in the silkworm, Bombyx mori L. J Invertebr Pathol 87: 8-15.

Kumar S, Tamura K, Nei M (2004) MEGA3: Integrated software for molecular evolutionary genetics analysis and sequence alignment. Brief Bioinform 5: 150-163.

Rami reddy K, Benchman K, Remadevi O (1992) Metabolic profiles of the haemolymph and fat body the silkworm, Bombyx mori, in response to parasitation by the uzifly Exorista sorbillans (DiptTachnidae), during the final instar. Sericologia 32: 227-233.

Ruderman NB, Saha AK, Vavvas D, Heydrick SJ, Kurowski TG (1997) Lipid abnormalities in muscle of insulin-resistant rodents. The malonyl CoA hypothesis. Ann NY Acad Sci USA 827: 221-230.

Scherer PE, Williams S, Fogliano M, Baldini G, Lodish HF (1995) A novel serum protein similar to C1q, produced exclusively in adipocytes. J Biol Chem 270: 26746-26749.

Thomas P, Tubbs C, Detweiler C, Das S, Ford L, Breckenridge-Miller D (2005) Binding characteristics, hormonal regulation and identity of the sperm membrane progestin receptor in Atlantic croaker. Steroids 70: 427-433.

Tomas E, Tsao TS, Saha AK, Murrey HE, Zhang Cc, Itani SI, Lodish HF, Ruderman NB (2002) Enhanced muscle fat oxidation and glucose transport by ACRP30 globular domain: acetyl-CoA carboxylase inhibition and AMP-activated protein kinase activation. Proc Natl Acad Sci USA 99: 16309-16313.
Tosca L, Froment P, Solnais P, Ferre P, Foufelle F, Dupont J (2005) Adenosine 5'-monophosphate-activated protein kinase regulates progesterone secretion in rat granulosa cells. Endocrinology 146: 4500-4513.

Tsuchida A, Yamauchi T, Ito Y, Hada Y, Maki T, Takekawa S, Kamon J, Kobayashi M, Suzuki R, Hara K, Kubota N, Terauchi Y, Froguel P, Nakae J, Kasuga M, Accili D, Tobe K, Ueki KR, Nagai R, Kadowaki T (2004) Insulin/Foxo1 pathway regulates expression levels of adiponectin receptors and adiponectin sensitivity. J Biol Chem 279: 30817-30822.

Wang J, Kean L, Yang J, Allan AK, Davies SA, Herzyk P, Dow JA (2004) Function-informed transcriptome analysis of Drosophila renal tubule. Genome Biol 5: R69.

Winder WW, Hardie DG (1999) AMP-activated protein kinase, a metabolic master switch: possible roles in type 2 diabetes. Am J Physiol 277: E1-10.

Xu JP, Chen KP, Yao Q, Liu MH, Gao GT, Zhao Y (2005) Identification and characterization of an NPV infectionrelated gene Bmsop2 in Bombyx mori L. J Appl Entomol 129: 425-431.

Yamauchi T, Kamon J, Ito Y, Tsuchida A, Yokomizo T, Kita S, Sugiyama T, Miyagishi M, Hara K, Tsunoda M, Murakami K, Ohteki T, Uchida S, Takekawa S, Waki H, Tsuno NH, Shibata Y, Terauchi Y, Froguel P, Tobe K, Koyasu S, Taira K, Kitamura T, Shimizu T, Nagai $\mathrm{R}$, Kadowaki T (2003) Cloning of adiponectin receptors that mediate antidiabetic metabolic effects. Nature 423 : 762-769.

Yamauchi T, Kamon J, Minokoshi Y, Ito Y, Waki H, Uchida S, Yamashita S, Noda M, Kita S, Ueki K, Eto K, Akanuma Y, Froguel P, Foufelle F, Ferre P, Carling D, Kimura S, Nagai R, Kahn BB, Kadowaki T (2002) Adiponectin stimulates glucose utilization and fatty-acid oxidation by activating AMP-activated protein kinase. Nat Med 8: 1288-1295. 